\title{
Analysis of right ventricular flow with 4-dimensional flow cardiovascular magnetic resonance imaging in patients with pulmonary arterial hypertension
}

\author{
Lei Wang ${ }^{1}$, Min Liu ${ }^{1}$, Pei Yao Zhang ${ }^{1}$, Jin Zhu Dai ${ }^{1}$, Hai Yi Ma ${ }^{1}$, Xin Cao Tao ${ }^{2}$, Wan Mu Xie ${ }^{2}$, Jun Wan ${ }^{2}$, \\ An Jing ${ }^{3}$
}

${ }^{1}$ Department of Radiology, China-Japan Friendship Hospital, Beijing, China; ${ }^{2}$ Department of Pulmonary and Critical Care Medicine, China-Japan Friendship Hospital, Beijing, China; ${ }^{3}$ Siemens Shenzhen Magnetic Resonance Ltd., Shenzhen, China

Correspondence to: Min Liu, MD. Department of Radiology, China-Japan Friendship Hospital, No. 2 Yinghua Dong Street, Hepingli, Chaoyang District, Beijing 100029, China. Email: drradiology@163.com.

Background: Cardiac flow closely interact with function, however, the correlation of right ventricular (RV) flow and function remains unknown, thus our objective is to observe right ventricular flow with four-dimensional phase-contrast cardiovascular magnetic resonance imaging (4D flow CMR) in patients with pulmonary arterial hypertension $(\mathrm{PAH})$ and to analyze flow components with RV function and hemodynamics.

Methods: This study retrospectively enrolled 30 patients with PAH (mean age: $49 \pm 13$ years, 16 females) and 14 age- and sex-matched healthy volunteers as controls (mean age: $44 \pm 12$ years, 9 females). All patients who underwent CMR and right heart catheterization (RHC) within 1 week between January 2019 and July 2020 were included. Hemodynamics were measured with RHC. RV flow components, including the percentages of direct flow (RVPDF), retained inflow (RVPRI), delayed ejection flow (RVPDEF) and residual volume (RVPRVo) were quantified using 4D flow CMR. The associations between RV flow components and other CMR metrics, clinical data, and hemodynamics were analyzed by Spearman's correlation analysis.

Results: In patients with PAH, RVPDF was decreased and RVPRVo was increased compared with the normal control group. The sum of RVPDF and RVPDEF RV was significantly correlated with RV ejection fraction (RVEF) $(\mathrm{r}=0.802, \mathrm{P}<0.001)$, and there was no notable difference between RVEF and the sum of RVPDF and RVPDEF ( $\mathrm{t}=0.251, \mathrm{P}=0.831$ ). Both RVPDF and RVPRVo were correlated (in opposite directions) with the RV end-diastolic volume index, RV end-systolic volume index, RV global longitudinal strain, and RVEF. RVPDF was negatively correlated with pulmonary vascular resistance (PVR), and positively correlated with cardiac output and cardiac index. RVPRVo was positively correlated with PVR and negatively correlated with cardiac output and cardiac index.

Conclusions: RV blood flow components qualified with 4D flow CMR is a valuable noninvasive method for the assessment of RV function and hemodynamics in patients with PAH.

Keywords: 4D flow cardiovascular magnetic resonance imaging (4D flow CMR); pulmonary arterial hypertension (PAH); right heart catheterization (RHC); right ventricular function; hemodynamics

Submitted Nov 15, 2020. Accepted for publication Apr 09, 2021.

doi: $10.21037 /$ qims-20-1267

View this article at: http://dx.doi.org/10.21037/qims-20-1267 


\section{Introduction}

Pulmonary arterial hypertension (PAH) is classified as pulmonary hypertension ( $\mathrm{PH})$ group $1(1)$, and is characterized by progressively increasing pulmonary vascular resistance (PVR) and right ventricular (RV) remodeling leading to RV failure. Although recent advances in therapeutic modalities have significantly improved the outcomes of patients with $\mathrm{PAH}$, the disorder still is associated with high rates of morbidity and mortality. A previous meta-analysis (2) showed RV ejection fraction (RVEF) to be the strongest predictor of mortality from PAH. From a pathophysiological perspective, since pulmonary arterial remodeling results in increased $\mathrm{RV}$ post-load and subsequently affects flow status in the right heart, RV structural and functional changes are preceded by changes in pulmonary arterial and RV flow $(3,4)$. Vortex formation in the main pulmonary artery has been shown to allow accurate estimation of pulmonary arterial pressure and the diagnosis of patients with PH (5); thus, intracardiac flow could be a more sensitive indicator for monitoring heart function. However, changes in RV flow and their correlation with hemodynamics and RV function in patients with $\mathrm{PAH}$ have not yet been elucidated.

Cardiovascular magnetic resonance (CMR) imaging is an accurate and reproducible method for the assessment of heart size, morphology, and function, and permits the noninvasive assessment of blood flow (6). Four-dimensional (3 spatial dimensions combined with time) phase-contrast (4D flow) CMR (7) can measure the velocity of blood flow in great vessels in any direction, and can also allow for visualization and quantification of the multidimensional intracardiac hemodynamic status and kinetic energy, as well as functional assessment without breath-hold and/or the use of contrast agents $(8,9)$. Stoll et al. used 4D flow CMR in patients with ischemic or dilated cardiomyopathy and observed a decrease in the volume and kinetic energy of direct flow (DF) compared with healthy controls (10). Elhawaz et al. (11) indicated that the kinetic energy of left ventricular (LV) blood flow was associated with the 6-minute walk test and LV remodeling after valvular intervention in patients with aortic stenosis. Fredriksson et al. (12) reported that RVDF had a larger volume and possessed a higher presystolic kinetic energy than the other flow components in healthy people. However, RV flow characteristics in patients with PAH have not yet been reported.

In the present study, we aimed to: (I) compare RV flow components between patients with $\mathrm{PAH}$ and healthy volunteers using 4D flow CMR; (II) evaluate the relationship between RV flow components and other functional metrics and serum biomarkers; and (III) assess the relationship between RV flow and hemodynamics.

\section{Methods}

\section{Study cobort and design}

This study was registered at the Chinese Clinical Trials Registry Center" (http://www.chictr.org/en/; registration number ChiCTR-OCH-14004929), and was approved by the institutional review board of China-Japan Friendship Hospital (IRB No. 2017-24). Informed consent was obtained from all participants or their family.

This study retrospectively enrolled patients with PAH from China-Japan Friendship Hospital. Patients who underwent right heart catheterization (RHC) and CMR within 1 week from January 2019 to July 2020 were included. The diagnoses of all patients were based on mean pulmonary arterial pressure (MPAP, $\geq 25 \mathrm{mmHg}$ ) and PVR [ $>3$ Wood units $(W U)$ ], as well as pulmonary capillary wedge pressure (PCWP, $\leq 15 \mathrm{mmHg}$ ) as assessed by RHC (1).

The included participants were aged between 18 and 65 years old. Patients with poor quality 4D flow or CMR images or incomplete RHC data were excluded. Patients in $\mathrm{PH}$ groups 2, 3, 4, and 5 were also excluded, as were those with cardiomyopathy, coronary stents, lung diseases, malignancies, diabetes mellitus, cirrhosis, kidney dysfunction, or hyperthyroidism. A normal control group comprising 14 age- and sex-matched healthy volunteers was also enrolled as the normal control group.

\section{RHC}

Hemodynamics were assessed by RHC. A Swan-Ganz standard thermodilution pulmonary artery catheter [Edwards (Shanghai) Medical Products Co., Ltd.] was placed at the right inferior pulmonary artery. The indices measured included right atrial pressure (RAP), MPAP, PCWP, and PVR. Cardiac output (CO) and the cardiac index $(\mathrm{CI})$ were determined using the Fick method.

\section{CMR examination}

All patients underwent CMR within 1 week of RHC. CMR was performed using a 1.5 Tesla clinical scanner (MAGNETOM Area, Siemens Healthcare, Erlangen, Germany) using an 18-channel phased-array surface coil. Images were acquired during end-expiratory breath- 
holds with retrospective electrocardiographic gating. First, standard cine long-axis 4-chamber and contiguous short axis slices covering both ventricles from base to apex were acquired with a balanced steady-state free precession sequence (typical acquisition parameters: repetition time/ echo time, 3.4/1.4 ms; flip angle, $50-60^{\circ}$; slice thickness, $6 \mathrm{~mm}$; in-plane spatial resolution, $1.8 \times 1.8 \mathrm{~mm}^{2}$; temporal resolution, $40 \mathrm{~ms} ; 25$ reconstructed cardiac phases).

Subsequently, 4D flow CMR images were acquired in the sagittal plane using a 3D retrospectively electrocardiographytriggered, navigator-gated prototype sequence with the following parameters: repetition time/echo time, 4.6/2.5 ms; flip angle, $7^{\circ}$; temporal resolution, $40 \mathrm{~ms}$; voxel size, $2.4 \times 2.4 \times 2.4 \mathrm{~mm}^{3}$; and velocity encoding, $100-150 \mathrm{~cm} / \mathrm{s}$. Twenty-five reconstructed cardiac phases were interpolated from 19-25 phases based on the patients' heart rate (ranging from 60 to 80 beats/min). Respiratory navigator gating was applied to minimize motion artifacts. Maxwell's correction and concomitant gradient field effects were corrected on the scanner. For each participant, the field of view was adjusted to cover the whole heart, and the scan time was 6-9 minutes.

\section{CMR analysis}

$\mathrm{RV}$ functional metrics, including the $\mathrm{RV}$ end-diastolic and end-systolic volume indexes (RVEDVI and RVESVI, respectively) and $R V$ and $L V$ ejection fractions (RVEF and LVEF, respectively), were analyzed using the cine sequence on Cardiac Function (SyngoVia workstation Siemens Healthcare, Erlangen, Germany) by a radiologist with 8 years' experience. A second radiologist with 6 years' experiences independently measured the RV global longitudinal strain (RVGLS), radial strain (RVGRS), and circumferential strain (RVGCS) by contouring the cine images at the end-diastolic phase using $\mathrm{CVI}^{42}$ (Version 5.11, Circle Cardiovascular Imaging Inc., Calgary, Alberta, Canada). RV flow components were also independently post-processed and analyzed using $\mathrm{CVI}^{42}$ (Version 5.11, Circle Cardiovascular Imaging Inc., Calgary, Alberta, Canada) by a radiologist with 5 years' experience.

The RV flow component analysis consisted of background phase correction, noise filtering, and velocity aliasing correction, as well as endocardial segmentation at end diastole and end systole with pathline generation from each segmented voxel. The positions of the pathlines (which are a form of visual simulation of a $3 \mathrm{D}$ velocity field that illustrates the travelling orbit of fluid particles in space at end systole) were divided into the following 4 functional flow components, which have been described previously (12): (I) DF, which refers to blood that enters the ventricle during diastole and leaves the ventricle during systole in the analyzed heartbeat; (II) delayed ejection flow (DEF), which describes blood that starts and resides inside the ventricle during diastole and leaves during systole in the analyzed heartbeat; (III) retained inflow (RI), which refers to blood that enters the ventricle during diastole but does not leave during systole in the analyzed heart beat; and (IV) residual volume (RVo), which describes blood that resides within the ventricle for at least 2 cardiac cycles. The percentage of each flow component in the $\mathrm{RV}$ was the ratio of each component volume (DF, DEF, RI and RVo) to RVEDV, including the percentage of DF (RVPDF), the percentage of DEF (RVPDEF), the percentage of RI (RVPRI), and the percentage of RVo (RVPRVo).

\section{Statistical analysis}

Statistical analyses were performed using SPSS 22 (Chicago, IL, USA). Data were expressed as mean \pm standard deviation (SD) or median with interquartile range (IQR). Nonparametric 2 independent samples $t$-tests were used to compare the 2 groups. Correlations were analyzed using Spearman's correlation. All tests were 2-sided, with $\mathrm{P}<0.05$ considered to indicate a statistically significant difference.

\section{Results}

\section{Baseline characteristics}

Thirty patients with PAH (mean age: $49.1 \pm 13.2$ years, 16 females) and 14 healthy volunteers (mean age: $43.7 \pm 12.3$ years, 9 females) were included in this study. The demographic and clinical data of all participants are shown in Table 1. There were no significant differences in sex, age, body mass index, heart rate, systolic blood pressure, or diastolic blood between the 2 groups. The respiratory rate of patients with $\mathrm{PAH}$ was faster than that of the healthy controls $(t=2.18, \mathrm{P}=0.035)$. Patients with $\mathrm{PAH}$ were all on at least $1 \mathrm{PAH}$-specific medication. One patient died of sudden massive hemoptysis during hospitalization, and the other patients are currently being followed up.

\section{$R V$ function, strain, and flow in PAH patients and bealthy controls}

The RV flows of participant patient with $\mathrm{PAH}$ and a normal control are visualized in Figure 1. As shown in 
Table 1 Demographic and clinical characteristics of all participants

\begin{tabular}{|c|c|c|c|c|}
\hline Characteristics & Total & $\mathrm{PAH}$ & Control & $\mathrm{P}$ \\
\hline Age (years) & $47.6 \pm 14.2$ & $49.1 \pm 13.2$ & $43.7 \pm 12.3$ & $\mathrm{t}=1.58(\mathrm{P}=0.121)$ \\
\hline $\operatorname{Sex}(F / M)$ & $25 / 19$ & $16 / 14$ & $9 / 5$ & $\chi^{2}=3.18(P=0.476)$ \\
\hline Respiratory rate (beats/min) & $20.2 \pm 1.4$ & $20.5 \pm 1.4$ & $19.6 \pm 1.1$ & $\mathrm{t}=2.18(\mathrm{P}=0.035)^{\star}$ \\
\hline Body Mass Index $\left(\mathrm{Kg} / \mathrm{m}^{2}\right)$ & $22.8 \pm 2.3$ & $23.1 \pm 2.4$ & $22.2 \pm 2.2$ & $\mathrm{t}=1.14(\mathrm{P}=2.262)$ \\
\hline Systolic BP (mmHg) & $116.1 \pm 14.9$ & $116.8 \pm 15.3$ & $114.7 \pm 14.2$ & $\mathrm{t}=0.42(\mathrm{P}=0.680)$ \\
\hline Diastolic BP (mmHg) & $79.5 \pm 13.3$ & $79.9 \pm 13.9$ & $78.7 \pm 12.3$ & $t=0.27(P=0.788)$ \\
\hline 6-minute walk distance (m) & $375(205,580)$ & $290(178,386)$ & $670(495,705)$ & $U=35.10(P<0.001)^{*}$ \\
\hline II & - & 12 & - & - \\
\hline III & - & 16 & - & - \\
\hline IV & - & 2 & - & - \\
\hline \multicolumn{5}{|l|}{ Serum biomarkers } \\
\hline NT-proBNP (pg/mL), median (IQR) & $322.5(68,975)$ & $548.0(265,1,500)$ & $34(23,83.5)$ & $\mathrm{U}=29.00(\mathrm{P}<0.001)^{*}$ \\
\hline CNTI (ng/mL), median (IQR) & $0.009(0.004,0.018)$ & $0.011(0.006,0.03)$ & $0.001(0,0.01)$ & $\mathrm{U}=75.00(\mathrm{P}<0.001)$ * \\
\hline \multicolumn{5}{|l|}{ CMR metrics } \\
\hline RVEDVI $\left(\mathrm{mL} / \mathrm{m}^{2}\right)$ & $94.2 \pm 38.2$ & $103.5 \pm 39.5$ & $66.4 \pm 13.3$ & $t=5.19(P<0.001)^{*}$ \\
\hline $\mathrm{RVCl}\left(\mathrm{L} / \mathrm{min} / \mathrm{m}^{2}\right)$ & $2.3 \pm 1.3$ & $2.1 \pm 1.4$ & $2.9 \pm 0.6$ & $\mathrm{t}=2.94(\mathrm{P}=0.005)^{\star}$ \\
\hline RVGRS (\%) & $12.5 \pm 7.6$ & $12.0 \pm 7.4$ & $14.8 \pm 7.8$ & $\mathrm{U}=140.00(\mathrm{P}=0.775)$ \\
\hline RVGCS (\%) & $-8.7 \pm 4.1$ & $-7.0 \pm 3.7$ & $-9.6 \pm 4.5$ & $\mathrm{U}=62.00(\mathrm{P}=0.038)^{*}$ \\
\hline RVGLS (\%) & $-14.5 \pm 7.4$ & $-11.9 \pm 6.6$ & $-22.2 \pm 3.6$ & $U=17.00(P<0.001)^{\star}$ \\
\hline RVPDF (\%) & $24.1 \pm 14.0$ & $16.4 \pm 9.2$ & $40.7 \pm 5.3$ & $t=-9.19(P<0.001)$ \\
\hline RVPDEF (\%) & $17.0 \pm 5.2$ & $15.8 \pm 5.9$ & $19.5 \pm 1.9$ & $t=-2.24(P=0.030)$ \\
\hline RVPRI (\%) & $17.4 \pm 5.4$ & $16.5 \pm 6.2$ & $19.1 \pm 2.1$ & $\mathrm{t}=-1.44(\mathrm{P}=0.157)$ \\
\hline RVPRVo (\%) & $41.1 \pm 19.6$ & $50.9 \pm 15.7$ & $20.3 \pm 5.3$ & $t=7.08(P<0.001)$ \\
\hline RVPDF + RVPDEF (\%) & $41.6 \pm 16.5$ & $32.1 \pm 11.5$ & $59.8 \pm 5.0$ & $t=-8.28(P<0.001)$ \\
\hline
\end{tabular}

Table 1 (continued) 
Table 1 (continued)

\begin{tabular}{lccc}
\hline Characteristics & Total & PAH & Control \\
\hline Right heart catheterization & & & P \\
Systolic PAP $(\mathrm{mmHg})$ & - & $76 \pm 23$ & - \\
Diastolic PAP $(\mathrm{mmHg})$ & - & $29 \pm 11$ & - \\
Mean PAP $(\mathrm{mmHg})$ & - & $48 \pm 14$ & - \\
PCWP $(\mathrm{mmHg})$ & - & $10 \pm 4$ & - \\
PVR $(\mathrm{WU})$ & - & $14.0 \pm 7.5$ & - \\
CO $(\mathrm{L} / \mathrm{min})$ & - & $3.0 \pm 1.2$ & - \\
$\mathrm{Cl}\left(\mathrm{L} / \mathrm{min} / \mathrm{m}^{2}\right)$ & - & $2.4 \pm 1.0$ & - \\
\hline
\end{tabular}

${ }^{*}, \mathrm{P}<0.05$. Values are expressed as mean $\pm \mathrm{SD}$ or number (percentage), or median and interquartile range (IQR). BP, blood pressure; $\mathrm{PH}$, pulmonary hypertension; NT-proBNP, N-terminal pro-brain natriuretic peptide; RVEDVI, right ventricular end-diastolic volume index; RVESVI, right ventricular end-systolic volume index; RVSVI, right ventricular stroke volume index; RVEF, right ventricular ejection fraction; RVMI, right ventricular myocardial mass index; RVGRS, right ventricular global radial strain; RVGCS, right ventricular global circumferential strain; RVGLS, right ventricular global longitudinal strain; RVPDF, the percentage of right ventricular direct flow; RVPDEF, the percentage of right ventricular delayed ejection flow; RVPRI, the percentage of right ventricular retained inflow; RVPRVo, the percentage of right ventricular residual volume; PAP, pulmonary arterial pressure; PCWP, pulmonary capillary wedge pressure; PVR, pulmonary vascular resistance; $\mathrm{CO}$, cardiac output; $\mathrm{Cl}$, cardiac index.

Table 1, compared with those in the control group, the RV functional metrics of the patients with $\mathrm{PAH}$, including $\mathrm{RV}$ end-diastolic volume index (RVEDVI), end-systolic volume index (RVESVI) and myocardial mass index (RVMI) were increased, while the RV stroke volume index (RVSVI), ejection fraction (RVEF), cardiac output (RVCO) and cardiac index (RVCI) were markedly reduced. Also, the RV global longitudinal strain (RVGLS) and RV global circumferential strain (RVGCS) were decreased in patients with PAH. As shown in Figure 2, RVPDF was significantly reduced in patients with $\mathrm{PAH}$ compared with the control group, whereas RVPRVo was markedly increased. However, RVPRI and RVPDFE were slightly lower in the PAH group than in the control group.

\section{Correlation between RV flow and function/serum biomarkers}

As shown in Table 2, RVPDF was negatively correlated with RVEDVI, RVESVI, RVMI and RVGLS, while being positively correlated with RVSVI, RVEF, RVCO and RVCI. Meanwhile, RVPRVo was positively correlated with RVEDVI, RVESVI, RVMI and RVGLS, but was negatively correlated with RVSVI, RVEF, RVCO and RVCI.

Figure 3 shows that the sum of RVPDF and RVPDEF (RVPDF + RVPDEF) was correlated with RVEF ( $r=0.802$,
$\mathrm{P}<0.001$ ), and there was no significant difference between $\mathrm{RVEF}$ and RVPDF + RVPDEF $(\mathrm{t}=0.251, \mathrm{P}=0.831)$. Figure 4 shows that RVPDF was positively correlated with the 6-minute walk distance $(6 \mathrm{MWD})(\mathrm{r}=0.612, \mathrm{P}<0.001)$ and was negatively correlated with serum $\mathrm{N}$-terminal pro-brain natriuretic peptide $(\mathrm{NT}$-proBNP) $(\mathrm{r}=-0.639, \mathrm{P}<0.001)$. Meanwhile, RVPRVo was positively correlated with NTproBNP $(r=0.637, \mathrm{P}=0.001)$ but was negatively correlated with the $6 \mathrm{MWD}(\mathrm{r}=-0.592, \mathrm{P}<0.001)$.

\section{Correlation between $R V$ flow components and bemodynamics in patients with $\mathrm{PAH}$}

Table 1 displays the study participants' hemodynamics, as measured by RHC. Table 3 shows the correlation between $\mathrm{RV}$ flow components and hemodynamics, as measured by RHC. We found that RVPDF was negatively correlated with PVR and positively correlated with CO and CI. Further, RVPRVo had positive correlations with RAP, MPAP and PVR, and negative correlations with CO and CI. None of the RV flow components were correlated with PCWP.

\section{Discussion}

This study has produced several findings. Firstly, compared to that of the healthy controls, the RVPDF was diminished 

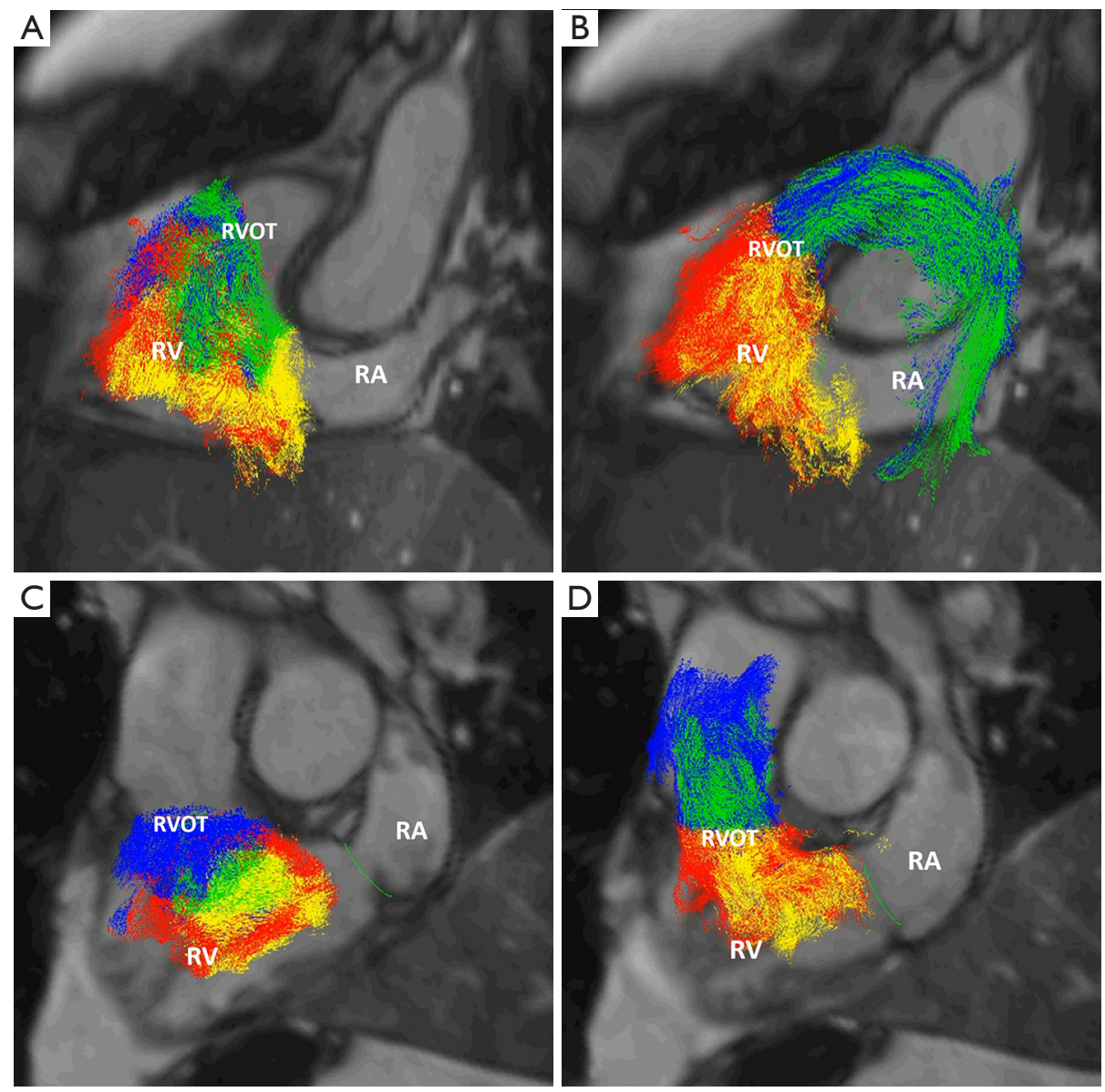

Figure 1 Right ventricular blood flow visualization in a normal participant (F/32 years old) (A,B) and a patient with idiopathic pulmonary artery hypertension (F/32 years old, mean pulmonary artery pressure: $40 \mathrm{mmHg}$ ) (C,D). (A,C) Pathline visualization of the right ventricular flow components (green represents DF; yellow represents retained inflow; blue represents DEF; and red represents residual volume) in diastole. (B,D) Pathline visualization of the RV flow components (green represents DF; yellow represents retained inflow; blue represents DEF; and red represents residual volume) in systole. RA, right atrium; RV, right ventricle; RVOT, right ventricular outflow tract.

in patients with PAH, while the RVPRVo was significantly increased. Secondly, RVPDF and RVPRVo were found to be closely correlated (in opposite directions) with the other RV functional metrics and RVGLS, while the RVEF was similar to the sum of RVPDF and RVPDEF. Thirdly, in patients with PAH, RVPDF and RVPRVo were closely correlated (in opposite directions) with MPAP, PVR, CO, and CI, which were also determined by RHC. To our knowledge, this is the first study to report the characteristics of RV flow and their correlation with RV function and hemodynamics in patients with $\mathrm{PAH}$.

In previous 4D flow MRI studies (12-14), the ventricular flow of normal controls was categorized into 4 components, and ventricular flow components and energetics were also characterized. DF refers to blood that enters the ventricle during diastole and leaves the ventricle during systole in the analyzed cardiac cycle. Among the 4 functional components of the LV diastolic volume, DF has the most direct route and the fastest transit through the $\mathrm{LV}$, and best preserves the kinetic energy from inflow to pre-systole (15). Both RVo and RI constitute the non-ejecting portion of ventricular volume. In our control group, the RVPDF and RVPRVo were slightly lower than previously published values from healthy individuals $(12,14)$. However, RVPDF was still the largest component among the 4 flow components, followed by RVPRVo, RVPRI, and RVPDEF.

Previous studies using 4D flow CMR $(14,16)$ indicated that RVPDF is reduced with the increased RV volumes in 
patients with mild ischemic heart disease, and that LVDF is also diminished with increased LV volumes. However, nonejecting flow was found to increase in patients with chronic ischemic heart disease. Similarly, compared to normal controls, decreased RVPDF and increased RVPRVo were prominent

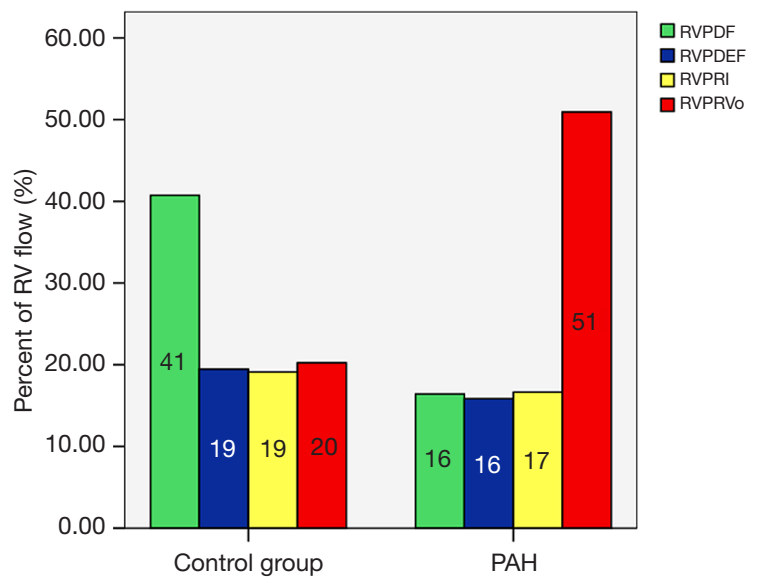

Figure 2 Comparison of right ventricular flow components between PAH patients and healthy controls. Compared with the control group, the percentage of RVPDF in patients with $\mathrm{PAH}$ is significantly reduced, while the percentage of RVPRVo is increased. RVPDF, right ventricular direct flow; RVPRVo, right ventricular residual volume.
RV flow characteristics in patients with $\mathrm{PAH}$, suggesting that changes in $4 \mathrm{D}$ flow-sspecific markers may detect RV dysfunction. DF possesses a significantly larger amount of kinetic energy than the other 3 flow components (17), while RVo, which describes blood that resides within the ventricle for at least 2 cardiac cycles, has the lowest kinetic energy among the 4 blood components.so, DF is prepared for effective systolic ejection.

Among four flow components, RVo had the largest volume in patients with $\mathrm{PAH}$. This means that the reduction in DF is insufficient for effective systolic ejection by virtue of preserved kinetic energy. In $\mathrm{PH}, \mathrm{RV}$ remodeling is initiated when the afterload increases (4), and the increase in RVo initially confers an advantage (e.g., acting as a buffer for kinetic energy redistribution), so as to reduce transfer of kinetic energy to potential energy that would result in the elevation of ventricular pressure. This situation is similar to LV blood flow in patients with left heart failure, and indicates that kinetic energy may be converted into less efficient configurations within the ventricle, resulting in the failure of any compensatory mechanism $(13,16)$. A decrease in DF may indicate $\mathrm{LV}$ dysfunction, even in subtle or subclinical LV remodeling. RVo has been shown to be an independent and significant index for distinguishing patients with atrial fibrillation from healthy controls (18).

$\mathrm{RV}$ function is the main predictor of survival in patients with PAH. In this research, RVPDF and RVPRVo were

Table 2 Correlation between right ventricular flow and functional data

\begin{tabular}{lcccc}
\hline Cardiac MR & RVPDF $(\%)$ & RVPDEF $(\%)$ & RVPRI $(\%)$ & RVPRVo $(\%)$ \\
\hline RVEDVI $\left(\mathrm{mL} / \mathrm{m}^{2}\right)$ & $-0.49(\mathrm{P}=0.001)$ & $-0.18(\mathrm{P}=0.270)$ & $-0.14(\mathrm{P}=0.382)$ & $0.41(\mathrm{P}=0.009)$ \\
RVESVI $\left(\mathrm{mL} / \mathrm{m}^{2}\right)$ & $-0.74(\mathrm{P}<0.001)^{\star}$ & $-0.39(\mathrm{P}=0.012)$ & $-0.33(\mathrm{P}=0.032)$ & $0.68(\mathrm{P}<0.001)^{\star}$ \\
RVSVI $\left(\mathrm{mL} / \mathrm{m}^{2}\right)$ & $0.34(\mathrm{P}=0.034)$ & $0.11(\mathrm{P}=0.489)$ & $0.06(\mathrm{P}=0.721)$ & $-0.28(\mathrm{P}=0.079)$ \\
RVMI $\left(\mathrm{g} / \mathrm{m}^{2}\right)$ & $-0.45(\mathrm{P}=0.004)$ & $-0.23(\mathrm{P}=0.154)$ & $-0.19(\mathrm{P}=0.259)$ & $0.49(\mathrm{P}=0.002)$ \\
RVEF $(\%)$ & $0.77(\mathrm{P}<0.001)^{\star}$ & $0.41(\mathrm{P}=0.008)$ & $0.37(\mathrm{P}=0.019)$ & $-0.73(\mathrm{P}<0.001)^{\star}$ \\
RVCO $(\mathrm{L} / \mathrm{min})$ & $0.58(\mathrm{P}<0.001)^{*}$ & $0.10(\mathrm{P}=0.552)$ & $0.56(\mathrm{P}=0.734)$ & $-0.41(\mathrm{P}=0.008)$ \\
RVCI $\left(\mathrm{L} / \mathrm{min} / \mathrm{m}^{2}\right)$ & $0.46(\mathrm{P}=0.004)$ & $0.12(\mathrm{P}=0.463)$ & $0.08(\mathrm{P}=0.610)$ & $-0.42(\mathrm{P}=0.007)$ \\
RVGRS $(\%)$ & $0.31(\mathrm{P}=0.055)$ & $0.11(\mathrm{P}=0.464)$ & $0.12(\mathrm{P}=0.444)$ & $-0.32(\mathrm{P}=0.042)$ \\
RVGCS $(\%)$ & $-0.08(\mathrm{P}=0.641)$ & $-0.06(\mathrm{P}=0.732)$ & $-0.09(\mathrm{P}=0.572)$ & $0.11(\mathrm{P}=0.509)$ \\
RVGLS $(\%)$ & $-0.62(\mathrm{P}<0.001)^{\star}$ & $-0.18(\mathrm{P}=0.277)$ & $-0.13(\mathrm{P}=0.414)$ & $0.57(\mathrm{P}<0.001)^{\star}$ \\
\hline
\end{tabular}

*, correlation is significant at the 0.001 level (2-tailed). RVEDVI, right ventricular end-diastolic volume index; RVESVI, right ventricular end-systolic volume index; RVSVI, right ventricular stroke volume index; RVEF, right ventricular ejection fraction; RVMI, right ventricular myocardial mass index; RVGRS, right ventricular global radial strain; RVGCS, right ventricular global circumferential strain; RVGLS, right ventricular global longitudinal strain; RVPDF, the percentage of right ventricular direct flow; RVPDEF, the percentage of right ventricular delayed ejection flow; RVPRI, the percentage of right ventricular retained inflow; RVPRVo, the percentage of right ventricular residual volume. 

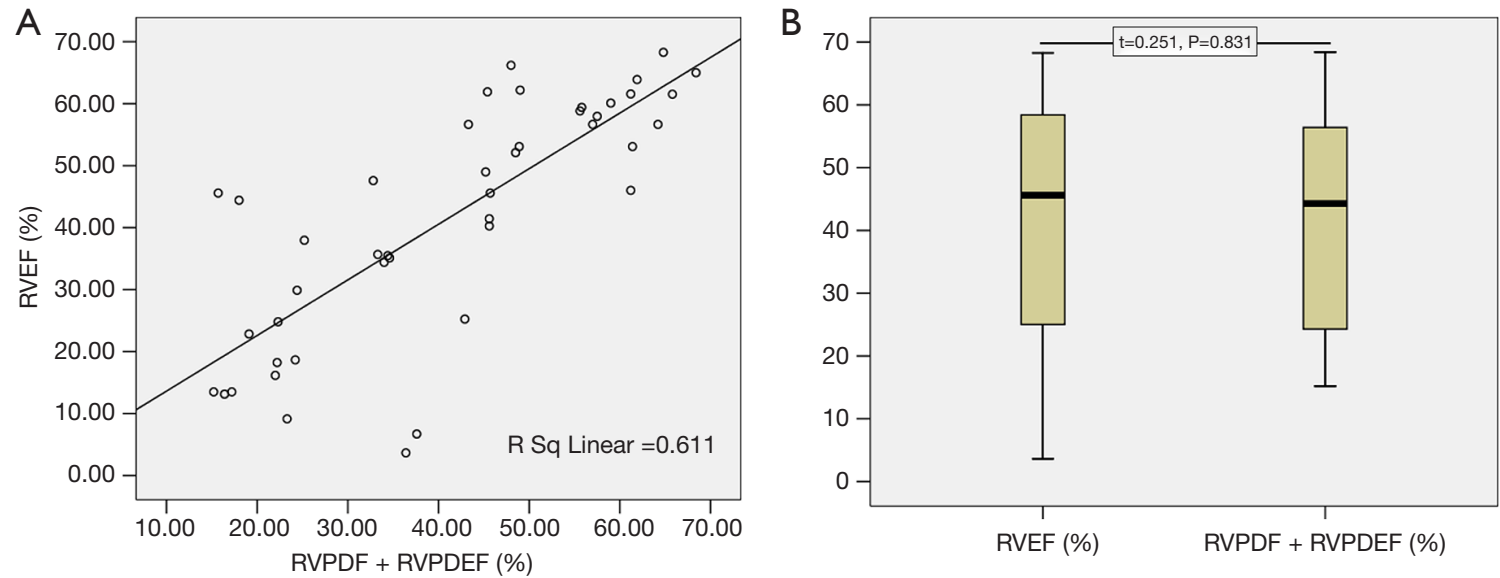

Figure 3 Correlation between right ventricular eject fraction (RVEF, as measured on cine images) and the sum of the percentage of RVPDF and (RVPDF + RVPDEF, as measured by 4D flow sequence). (A) RVEF is correlated with the sum of RVPDF and RVPDEF (RVPDF + RVPDEF); (B) RVEF is comparable to the sum of RVPDF and RVPDEF (RVPDF + RVPDEF). RVPDF, right ventricular direct flow; RVPDEF, right ventricular delayed ejection flow.
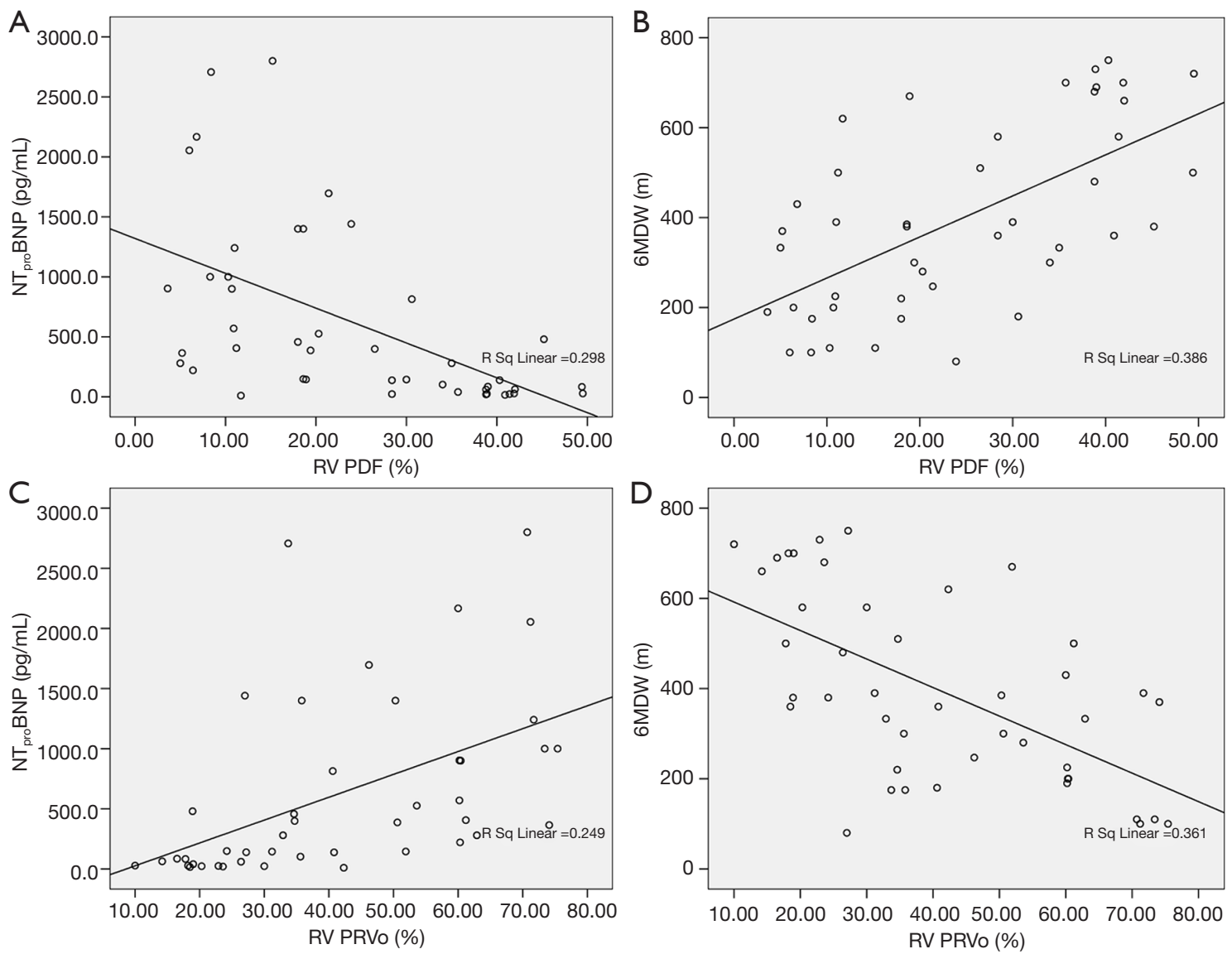

Figure 4 Correlation between RV flow and clinical markers. (A) RVPDF is negatively correlated with the serum NT-proBNP; (B) RVPDF is positively correlated with the 6MWD; (C) PRVo is positively correlated with NT-proBNP; (D) PRVo is negatively correlated with 6MWD. NT-proBNP, N-terminal pro-brain natriuretic peptide; 6MWD, 6-minute walk distances. 
Table 3 Correlation between right ventricular flow and hemodynamics in patients with PAH

\begin{tabular}{lcccccc}
\hline RV flow components & RAP $(\mathrm{mmHg})$ & MPAP $(\mathrm{mmHg})$ & $\mathrm{PAWP}(\mathrm{mmHg})$ & $\mathrm{PVR}(\mathrm{WU})$ & $\mathrm{CO}(\mathrm{L} / \mathrm{min})$ & $\mathrm{Cl}\left(\mathrm{L} / \mathrm{min} / \mathrm{m}^{2}\right)$ \\
\hline RVPDF $(\%)$ & $-0.51(\mathrm{P}=0.002)$ & $-0.45(\mathrm{P}=0.005)$ & $0.21(\mathrm{P}=0.207)$ & $-0.74(\mathrm{P}<0.001)^{\star}$ & $0.66(\mathrm{P}<0.001)^{\star}$ & $0.64(\mathrm{P}<0.001)^{\star}$ \\
RVPDEF (\%) & $-0.45(\mathrm{P}=0.007)$ & $-0.30(\mathrm{P}=0.053)$ & $0.16(\mathrm{P}=0.352)$ & $-0.32(\mathrm{P}=0.072)$ & $0.20(\mathrm{P}=0.272)$ & $0.16(\mathrm{P}=0.380)$ \\
RVPRI (\%) & $-0.44(\mathrm{P}=0.007)$ & $-0.36(\mathrm{P}=0.027)$ & $0.16(\mathrm{P}=0.335)$ & $-0.35(\mathrm{P}=0.049)$ & $0.25(\mathrm{P}=0.163)$ & $0.20(\mathrm{P}=0.275)$ \\
RVPRVo (\%) & $0.57(\mathrm{P}<0.001)^{\star}$ & $0.56(\mathrm{P}<0.001)^{\star}$ & $0.19(\mathrm{P}=0.256)$ & $0.73(\mathrm{P}<0.001)^{\star}$ & $-0.56(\mathrm{P}<0.001)^{\star}$ & $-0.54(\mathrm{P}<0.001)^{\star}$ \\
\hline
\end{tabular}

*, correlation is significant at the 0.001 level (2-tailed). RVPDF, the percentage of right ventricular direct flow; RVPDEF, the percentage of right ventricular delayed ejection flow; RVPRI, the percentage of right ventricular retained inflow; RVPRVo, the percentage of right ventricular residual volume; RAP, mean right atrial pressure, MPAP, mean pulmonary arterial pressure; PAWP, pulmonary capillary wedge pressure; PVR, pulmonary vascular resistance; $\mathrm{CO}$, cardiac output; $\mathrm{Cl}$, cardiac index.

correlated (in opposite directions) with other RV functional metrics, such as RVEF, thus confirming that ventricular flow status and function are highly interdependent on 1 side. The significant reduction of RVPDF and elevation of RVPRVo accompanied by a slight increase in RVPRI and RVPDEF, which is a phenomenon in PAH, suggests that $\mathrm{PDF}$ and PRVo can serve as indicators of RV dysfunction. Moreover, the sum of RVPDF and RVPDEF was similar to the RVEF, which was calculated using steady-state free precession cine images, indicating that 4D flow provides ventricular blood flow status information to enable an understanding of $\mathrm{RV}$ dysfunction without compensating for respiratory motion $(19,20)$. It can also be used to calculate RVEF, which is the sum of RVPDF and RVPDEF, without the need for the breath-holding requirements used for conventional cine images.

Strain has been widely used to evaluate ventricular deformation and mechanics. In a previous study (21), global longitudinal strain, global circumferential stain, and global radial strain were reduced in patients with $\mathrm{PH}$ and RVEF $<50 \%$. In the present study, we found that global longitudinal strain and global circumferential strain were decreased in patients with PAH; only global longitudinal strain was correlated with RVPDF and RVPRVo (in opposite directions). Although the detailed mechanism is unclear, our data confirms the close interaction between morphology, function, and blood flow. The 6MWD and NT-proBNP are used to conduct risk assessments of patients with $\mathrm{PAH}$, and may also provide prognostic information and thus be used to guide therapeutic decisions (1). Both RVPDF and RVPRVo were correlated with the $6 \mathrm{MWD}$ and NTproBNP, suggesting that RV flow component analysis can provide prognostic information. However, the cutoff value for risk assessment is still unknown. Previous studies have reported that $4 \mathrm{D}$ flow $\mathrm{CMR}$ could provide a non-invasive estimate of PVR and MPAP (22-24). We further observed that RVPDF and RVPRVo were inversely correlated with PVR and MPAP. To our knowledge, the current work is the first to demonstrate the correlation between RV flow components and hemodynamics. Ventricular structures and blood flow are highly interdependent; thus, as the ventricular flow is altered in the early stages of remodeling, flow itself can influence disease progression.

\section{Study limitations}

There are several limitations in this study that should be noted. Firstly, the current results only relate to a relatively small number of sex- and age-matched patients and volunteers. Changes to ventricular flow related to age, sex, respiratory, and heart rate remain unknown. Also, our study only included patients with $\mathrm{PAH}$; whether the current findings can be extrapolated to other etiologies of $\mathrm{PH}$ remains unclear. Thirdly, since most patients were New York Heart Association classification II/III, further study is required on ventricular flow in the assessment of subtle right ventricular dysfunction. Moreover, kinetic energy of flow components is known to be predictive of patients' functional capacity; however, due to software algorithm limitations, we did not analyze the kinetic energy of each flow component. Therefore, the kinetic energy of RV flow in PAH should be further investigated in future research. Finally, the lack of longitudinal data limited the assessment of the prognostic significance of the 4D flow-specific parameters for clinical outcomes, and future follow-up studies are needed.

\section{Conclusions}

This study has demonstrated that 4D flow CMR can permit the visualization and quantification of RV flow components. 
RVDF is significantly reduced and residual volumes are markedly increased in patients with PAH. RV blood flow components are closely correlated with RV function and hemodynamics in patients with PAH. We suggest that 4D flow imaging is included in the CMR protocol in the evaluation of patients with $\mathrm{PAH}$.

\section{Acknowledgments}

We would like to acknowledge Jumatay Biekan (Circle Cardiovascular Imaging, Calgary) for his assistance in $4 \mathrm{D}$ flow MRI image post-processing.

Funding: This work was supported by the Beijing Nature Science Foundation (grant no. 7182149), the National Natural Science Foundation of China (grant no. 81871328), the Youth Talents project of Chinese Academy of Medical Science (grant no. 2018RC320013), and the Beijing Science and Technology Commission Pharmaceutical and Technology Innovation Project (grant no. Z181100001918034).

\section{Footnote}

Conflicts of Interest: All authors have completed the ICMJE uniform disclosure form (available at http://dx.doi. org/10.21037/qims-20-1267). The authors have no conflicts of interest to declare.

Ethical Statement: The authors are accountable for all aspects of the work in ensuring that questions related to the accuracy or integrity of any part of the work are appropriately investigated and resolved. This study was approved by the ethics committee of China-Japan Friendship Hospital (IRB No. 2017-24), and was performed according to the latest version of the Declaration of Helsinki (as revised in 2013) for Medical Research involving Human Subjects. Informed consent was obtained from all patients.

Open Access Statement: This is an Open Access article distributed in accordance with the Creative Commons Attribution-NonCommercial-NoDerivs 4.0 International License (CC BY-NC-ND 4.0), which permits the noncommercial replication and distribution of the article with the strict proviso that no changes or edits are made and the original work is properly cited (including links to both the formal publication through the relevant DOI and the license). See: https://creativecommons.org/ licenses/by-nc-nd/4.0/.

\section{References}

1. Galiè N, Humbert M, Vachiery JL, Gibbs S, Lang I, Torbicki A, et al, International Society for Heart and Lung Transplantation (ISHLT). Eur Heart J 2016;37:67-119.

2. Baggen VJ, Leiner T, Post MC, van Dijk AP, RoosHesselink JW, Boersma E, Habets J, Sieswerda GT. Cardiac magnetic resonance findings predicting mortality in patients with pulmonary arterial hypertension: a systematic review and meta-analysis. Eur Radiol 2016;26:3771-80.

3. Mendes-Ferreira P, Santos-Ribeiro D, Adão R, MaiaRocha C, Mendes-Ferreira M, Sousa-Mendes C, LeiteMoreira AF, Brás-Silva C. Distinct right ventricle remodeling in response to pressure overload in the rat. Am J Physiol Heart Circ Physiol 2016;311:H85-95.

4. van der Bruggen CEE, Tedford RJ, Handoko ML, van der Velden J, de Man FS. RV pressure overload: from hypertrophy to failure. Cardiovasc Res 2017;113:1423-32.

5. Reiter G, Reiter U, Kovacs G, Olschewski H, Fuchsjager $M$. Blood flow vortices along the main pulmonary artery measured with MR imaging for diagnosis of pulmonary hypertension. Radiology 2015;275:71-9.

6. Peacock AJ, Vonk Noordegraaf A. Cardiac magnetic resonance imaging in pulmonary arterial hypertension. Eur Respir Rev 2013;22:526-34.

7. Azarine A, Garçon P, Stansal A, Canepa N, Angelopoulos G, Silvera S, Sidi D, Marteau V, Zins M. Four-dimensional Flow MRI: Principles and Cardiovascular Applications. Radiographics 2019;39:632-48.

8. Schäfer M, Ivy DD, Abman SH, Stenmark K, Browne LP, Barker AJ, Mitchell MB, Morgan GJ, Wilson N, Shah A, Kollengode M, Naresh N, Fonseca B, DiMaria M, Buckner JK, Hunter KS, Kheyfets V, Fenster BE, Truong U. Differences in pulmonary arterial flow hemodynamics between children and adults with pulmonary arterial hypertension as assessed by 4D-flow CMR studies. Am J Physiol Heart Circ Physiol 2019;316:H1091-104.

9. Hu L, Ouyang R, Sun A, Wang Q, Guo C, Peng Y, Qin Y, Zhang Y, Xiang Y, Zhong Y. Pulmonary artery hemodynamic assessment of blood flow characteristics in repaired tetralogy of Fallot patients versus healthy child volunteers. Quant Imaging Med Surg 2020;10:921-33.

10. Stoll VM, Hess AT, Rodgers CT, Bissell MM, Dyverfeldt P, Ebbers T, Myerson SG, Carlhäll CJ, Neubauer S. 
Left Ventricular Flow Analysis. Circ Cardiovasc Imaging 2019;12:e008130.

11. Elhawaz A, Archer CT, Zafar H, Fidock B, Barker N, Jones R, Rothman A, Hose R, Al-Mohammad A, Briffa N, Hunter S, Braidley P, Hall IR, Grech E, van der Geest RJ, Gunn JP, Swift AJ, Wild JM, Garg P. Left ventricular blood flow kinetic energy is associated with the six-minute walk test and left ventricular remodelling post valvular intervention in aortic stenosis. Quant Imaging Med Surg 2021;11:1470-82.

12. Fredriksson AG, Zajac J, Eriksson J, Dyverfeldt P, Bolger AF, Ebbers T, Carlhäll CJ. 4-D blood flow in the human right ventricle. Am J Physiol Heart Circ Physiol 2011;301:H2344-50.

13. Eriksson J, Carlhäll CJ, Dyverfeldt P, Engvall J, Bolger AF, Ebbers T. Semi-automatic quantification of 4D left ventricular blood flow. J Cardiovasc Magn Reson 2010;12:9.

14. Fredriksson AG, Svalbring E, Eriksson J, Dyverfeldt P, Alehagen U, Engvall J, Ebbers T, Carlhäll CJ. 4D flow MRI can detect subtle right ventricular dysfunction in primary left ventricular disease. J Magn Reson Imaging 2016;43:558-65.

15. Bolger AF, Heiberg E, Karlsson M, Wigström L, Engvall J, Sigfridsson A, Ebbers T, Kvitting JP, Carlhäll CJ, Wranne B. Transit of blood flow through the human left ventricle mapped by cardiovascular magnetic resonance. J Cardiovasc Magn Reson 2007;9:741-7.

16. Svalbring E, Fredriksson A, Eriksson J, Dyverfeldt P, Ebbers T, Bolger AF, Engvall J, Carlhäll CJ. Altered Diastolic Flow Patterns and Kinetic Energy in Subtle Left Ventricular Remodeling and Dysfunction Detected by 4D Flow MRI. PLoS One 2016;11:e0161391.

17. Carlsson M, Heiberg E, Toger J, Arheden H. Quantification of left and right ventricular kinetic energy using four-dimensional intracardiac magnetic resonance imaging flow measurements. Am J Physiol Heart Circ Physiol 2012;302:H893-900.

Cite this article as: Wang L, Liu M, Zhang PY, Dai JZ, Ma HY, Tao XC, Xie WM, Wan J, Jing A. Analysis of right ventricular flow with 4-dimensional flow cardiovascular magnetic resonance imaging in patients with pulmonary arterial hypertension. Quant Imaging Med Surg 2021;11(8):3655-3665. doi: 10.21037/qims-20-1267
18. Kim H, Sheitt H, Jamalidinan F, Wilton S, White J, Garcia J. Left Ventricular Flow Analysis in Atrial Fibrillation. Annu Int Conf IEEE Eng Med Biol Soc $2020 ; 2020: 1182-5$.

19. Uribe S, Beerbaum P, Sorensen TS, Rasmusson A, Razavi R, Schaeffter T. Four-dimensional (4D) flow of the whole heart and great vessels using real-time respiratory selfgating. Magn Reson Med 2009;62:984-92.

20. Kanski M, Töger J, Steding-Ehrenborg K, Xanthis C, Bloch KM, Heiberg E, Carlsson M, Arheden H. Whole-heart four-dimensional flow can be acquired with preserved quality without respiratory gating, facilitating clinical use: a head-to-head comparison. BMC Med Imaging 2015;15:20.

21. de Siqueira ME, Pozo E, Fernandes VR, Sengupta PP, Modesto K, Gupta SS, Barbeito-Caamaño C, Narula J, Fuster V, Caixeta A, Sanz J. Characterization and clinical significance of right ventricular mechanics in pulmonary hypertension evaluated with cardiovascular magnetic resonance feature tracking. J Cardiovasc Magn Reson 2016;18:39.

22. Schäfer M, Barker AJ, Kheyfets V, Stenmark KR, Crapo J, Yeager ME, Truong U, Buckner JK, Fenster BE, Hunter KS. Helicity and Vorticity of Pulmonary Arterial Flow in Patients with Pulmonary Hypertension: Quantitative Analysis of Flow Formations. J Am Heart Assoc 2017;6:e07010.

23. Odagiri K, Inui N, Hakamata A, Inoue $Y$, Suda T, Takehara Y, Sakahara H, Sugiyama M, Alley MT, Wakayama T, Watanabe H. Non-invasive evaluation of pulmonary arterial blood flow and wall shear stress in pulmonary arterial hypertension with $3 \mathrm{D}$ phase contrast magnetic resonance imaging. Springerplus 2016;5:1071.

24. Reiter U, Reiter G, Kovacs G, Stalder AF, Gulsun MA, Greiser A, Olschewski H, Fuchsjäger M. Evaluation of elevated mean pulmonary arterial pressure based on magnetic resonance 4D velocity mapping: comparison of visualization techniques. PLoS One 2013;8:e82212. 